CORRECTION

https://doi.org/10.1038/s41586-019-1628-y

\title{
Author Correction: Human placenta has no microbiome but can contain potential pathogens
}

Marcus C. de Goffau, Susanne Lager, Ulla Sovio, Francesca Gaccioli, Emma Cook, Sharon J. Peacock, Julian Parkhill, D. Stephen Charnock-Jones \&

Gordon C. S. Smith

Correction to: Nature https://doi.org/10.1038/s41586-019-1451-5, published online 31 July 2019.

In this Article, the Supplementary Information file containing the $16 \mathrm{~S}$ rRNA sequencing results has been adapted to include a second tab labelled 'ENA link' that provides direct links between individual raw data files in the European Nucleotide Archive (ENA) and the sample numbers used in the Article. This has been added to make it easier for researchers to retrieve 16S rRNA gene datasets from the ENA and link these with the relevant metadata; previously, it was possible to link the ENA files to the corresponding sample via the cram file names, but this was overly complicated and the relationships were not obvious.

In addition, in the Source Data files for Fig. 1 and Extended Data Fig. 5, the arbitrary numbers given to the placental samples did not correspond to the same samples in cohorts 1 and 2. Matching sample numbers have now been provided in both Source Data files, and have also been added to the Supplementary Information sequencing file. Two minor errors in the headings in the Source Data file for Extended Data Fig. 5 (cells F3 and AM1) have also been corrected. We also noticed that the wrong file had been provided as Source Data for Fig. 3 and this has now been replaced. For transparency to readers, the original Supplementary Information sequencing file and the original Source Data files for Figs. 1, 3 and Extended Data Fig. 5 are provided as Supplementary Information to this Amendment.

Finally, a minor error in the name of the tab in the Source Data file for Extended Data Fig. 7 (originally named 'Extended Data Fig. 5') has also been corrected.

All of these additions and corrections do not change any of the data or conclusions reported in the Article, and are only necessary to facilitate reanalysis of our data by other researchers.

Supplementary information is available in the online version of this Amendment. 